\title{
Allosteric inhibition of CRISPR-Cas9 by bacteriophage-derived peptides
}

Yan-ru Cui 1,2,3,4†, Shao-jie Wang ${ }^{1 \dagger}$, Jun Chen ${ }^{5}$, Jie Li 1,2,3,4 Wenzhang Chen', Shuyue Wang ${ }^{1,2,3,4}$, Bing Meng ${ }^{1}$, Wei Zhu' ${ }^{1}$ Zhuhong Zhang ${ }^{6}$, Bei Yang ${ }^{1}$, Biao Jiang ${ }^{1}$, Guang Yang ${ }^{1}$, Peixiang Ma ${ }^{1 *}$ (D) and Jia Liu ${ }^{1 *}$ (D)

\begin{abstract}
Background: CRISPR-Cas9 has been developed as a therapeutic agent for various infectious and genetic diseases. In many clinically relevant applications, constitutively active CRISPR-Cas9 is delivered into human cells without a temporal control system. Excessive and prolonged expression of CRISPR-Cas9 can lead to elevated off-target cleavage. The need for modulating CRISPR-Cas9 activity over time and dose has created the demand of developing CRISPR-Cas off switches. Protein and small molecule-based CRISPR-Cas inhibitors have been reported in previous studies.

Results: We report the discovery of Cas9-inhibiting peptides from inoviridae bacteriophages. These peptides, derived from the periplasmic domain of phage major coat protein G8P (G8P PD), can inhibit the in vitro activity of Streptococcus pyogenes Cas9 (SpCas9) proteins in an allosteric manner. Importantly, the inhibitory activity of G8P PD on SpCas9 is dependent on the order of guide RNA addition. Ectopic expression of full-length G8P (G8P $\mathrm{FL}_{\mathrm{L}}$ ) or G8P $\mathrm{PD}$ in human cells can inactivate the genome-editing activity of SpyCas 9 with minimum alterations of the mutation patterns. Furthermore, unlike the anti-CRISPR protein Acrll4A that completely abolishes the cellular activity of CRISPR-Cas9, G8P co-transfection can reduce the off-target activity of co-transfected SpCas9 while retaining its on-target activity.
\end{abstract}

Conclusion: G8Ps discovered in the current study represent the first anti-CRISPR peptides that can allosterically inactivate CRISPR-Cas9. This finding may provide insights into developing next-generation CRISPR-Cas inhibitors for precision genome engineering.

Keywords: CRISPR-Cas9, Inoviridae bacteriophage, Major coat protein G8P, Allosteric inhibition, Off-target activity

\section{Background}

Clustered Regularly Interspaced Short Palindromic Repeats (CRISPR) is the bacterial adaptive immune system to defend bacteriophage infections [1-3]. During infection, invader DNA is captured and integrated into bacterial genome as CRISPR array. Sequences from CRISPR array are transcribed and processed into CRISPR RNAs (crRNAs), which direct CRISPR-associated (Cas) proteins to foreign nucleic acids [1, 2]. Type II CRISPR-Cas systems function with streamlined components comprising of a single nuclease protein such as Cas9 [4]. The modular and programmable features make CRISPR-Cas9 one of the most widely used tools for genome engineering applications [5-8]. However, CRISPR-Cas9

\footnotetext{
* Correspondence: mapx@shanghaitech.edu.cn; liujia@shanghaitech.edu.cn

†Yan-ru Cui and Shao-jie Wang contributed equally to this work.

'Shanghai Institute for Advanced Immunochemical Studies, ShanghaiTech

University, Shanghai 201210, China

Full list of author information is available at the end of the article
}

is associated with off-target cleavage [9], chromosomal rearrangement [10], and genotoxicity [11]. These side effects mainly arise from the excessive or prolonged expression of CRISPR-Cas9 [12-14]. As a therapeutic agent, CRISPR-Cas9 is often constitutively expressed in host cells [15], making the elevated off-activity a major safety concern. Temporal control of SpCas9 activity has been investigated as an approach to improving its specificity in human cells. Technologies enabling the temporal control of CRISPR-Cas9 include optogenetic tools, intein splicing system, small molecule inducers or inhibitors [16-20], and anti-CRISPR proteins (Acrs) [21, 22].

The most investigated CRISPR-Cas inhibitors are the naturally occurring, phage-derived Acrs. Bacteriophages can use Acrs to antagonize the CRISPR-Cas immunity in bacteria [23, 24]. A number of Acrs have been identified for type I [23, 25-28], type II [29-32], and type V [28, 33] CRISPR-Cas systems. Acrs can be adapted to regulate CRISPR-Cas activities in bacteria [34], yeast [35], and 
mammalian cells [29, 31, 34, 36-38]. Biosensor [39] and synthetic circuits [40] can be devised based on Acrcoupled CRISPR-Cas systems. Moreover, Acrs can be harnessed to enable the temperature-responsive [41] and optogenetic [42] control of CRISPR-Cas activity. Importantly, Acrs can enhance the editing [38] and cell-type [43] specificities and reduce the cytotoxicity [11] of CRISPRCas-mediated genome editing. It has also been reported that Acrs can facilitate the production of CRISPR-carrying viral vectors by restricting CRISPR self-cleavage [44].

Currently known Acrs inhibit CRISPR-Cas systems by interfering with Cas protein-mediated DNA surveillance or cleavage [21]. For instance, AcrIIA4 mimics doublestranded DNA (dsDNA) and occupies the protospacer adjacent motif (PAM) recognition site of SpCas9, thereby preventing Cas9 protein from binding to the target DNA [38, 45, 46]. Using a different mechanism, AcrIIC3 perturbs DNA binding by inducing the dimerization of Cas9 protein [36]. An alternative Cas inactivation strategy by Acrs is to interact with DNAbound Cas proteins and block subsequent DNA cleavage, as seen with AcrF3 [47, 48] and AcrIIC1 [36]. In addition, some Acrs can function as acetyltransferase and inactivate CRISPR-Cas activity by post-translational modifications [49]. Different Acrs may inactivate CRISPR-Cas via identical mechanisms while possessing low sequence similarities [21]. The Acrs characterized to date share no common sequence motifs except for a putative transcriptional element referred to as antiCRISPR-associated genes (Acas) that are commonly found downstream of the Acr genes in the bacteriophage genome [26]. The poorly understood sequencestructure-activity relationship largely hampers the systemic discovery of novel Acrs. In addition to proteinbased inhibitors, small-molecule CRISPR-Cas inhibitors have been developed using a high-throughput screening platform [20]. These small molecules are cell-permeable and can reversely disrupt SpCas9-DNA interaction, thus enabling dose and temporal control of SpCas9. However, relatively high concentrations of $10 \mu \mathrm{M}$ or above are required for small molecules to achieve efficient inhibition [20].

Along with small molecules and proteins, peptides represent an alternative class of CRISPR-inhibiting agents with distinct biochemical features. In this study, we report the discovery of Cas9-inactivating peptides from inoviridae bacteriophages. In an attempt to develop anti-CRISPR antibodies using well established phage display technology [50], we are surprised to find that the commonly used laboratory bacteriophage strain M13 served as a source of Cas9-inactivating agent. Subsequent analyses showed that the periplasmic domain of the major coat protein G8P (G8P $\mathrm{PD}_{\mathrm{PD}}$ ) from several inoviridae bacteriophages, which contain M13 phage, inhibited the in vitro and in vivo activity of SpCas9 in an allosteric manner. Our study hence expands the inhibitor toolbox for the temporal control of CRISPR-Cas activity.

\section{Results \\ Intact M13 phage inhibits the in vitro DNA cleavage activity of SpCas9}

In a conventional phage display experiment, we surprisingly discovered that intact M13 phage [51] itself could block the DNA cleavage activity of purified SpCas9 proteins in a dose-dependent manner with an approximate half maximum inhibitory concentration $\left(\mathrm{IC}_{50}\right)$ of $5 \mathrm{nM}$ (Fig. 1a), which corresponds to a phage titer of $3 \times 10^{9}$ $\mathrm{PFU} / \mu \mathrm{L}$. Interestingly, phage-mediated SpCas 9 inactivation occurred only if phage was supplemented to the reaction prior to the addition of sgRNA, but not post the formation of SpCas9-sgRNA ribonucleoproteins (RNPs) (Fig. 1b). The order-of-addition-dependent inhibition suggested that competition for sgRNA-binding site in SpCas9 is a possible mechanism of the inhibitory activity of M13 phage.

\section{M13 phage major coat protein-derived peptide inhibits the in vitro DNA cleavage activity of SpCas9}

Next we sought to determine the components in M13 phage that contribute to SpCas9 inactivation. M13 phage has a simple, compact genome comprising of 11 protein-coding sequences. Considering the accessibility, surface-exposed phage proteins most likely serve as the sources of Cas9-inhibiting agents. Fully packaged M13 phage contains approximately 2700 copies of major coat protein pVIII (G8P) and 5 copies each of minor coat proteins pIII, pVI, pVII, and pXI [52-54]. We initiated our investigation on the major coat protein G8P due to its abundance on M13 phage surface. The 73-amino acid major coat protein G8P contains four segments including signal peptide, periplasmic domain, transmembrane helix, and cytoplasmic domain (Fig. 1c). After G8P maturation, signal peptide is cleaved and transmembrane helix is inserted into phage membrane, leaving periplasmic domain the only region on phage surface. Therefore, we synthesized a 21-amino acid peptide constituting the periplasmic domain of G8P (G8P $\mathrm{PD}$ ) and examined its inhibitory activity on SpCas9.

We found that G8P $P_{\mathrm{PD}}$ inhibited the activity of SpCas9 with an approximate $\mathrm{IC}_{50}$ of $5 \mu \mathrm{M}$ (Fig. 1d), which was 1000 -fold lower than that of intact M13 phage. Similar to the intact phage, G8P $\mathrm{P}_{\mathrm{PD}}$ suppressed the in vitro DNA cleavage of SpCas9 in an order-of-addition-dependent manner (Fig. 1e), indicating that G8P $\mathrm{P}_{\mathrm{PD}}$ may inactivate SpCas9 by specifically interfering with apo-SpCas9.

To explore whether peptides with sequence similarity to $\mathrm{GPP}_{\mathrm{PD}}$ can inhibit Cas9 activity, we used Blast to search for peptide homologs of M13 G8P $\mathrm{PD}_{\mathrm{PD}}$. We identified several peptide sequences from inoviridae phages 

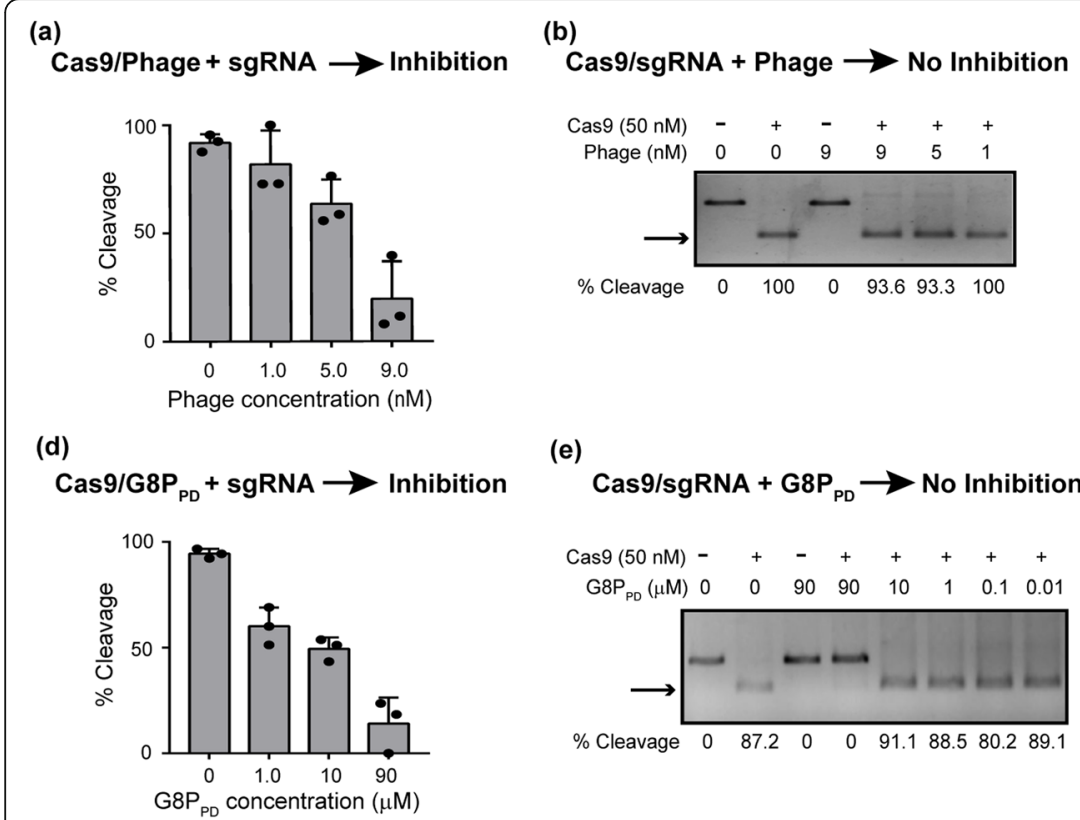

(c)

(e)
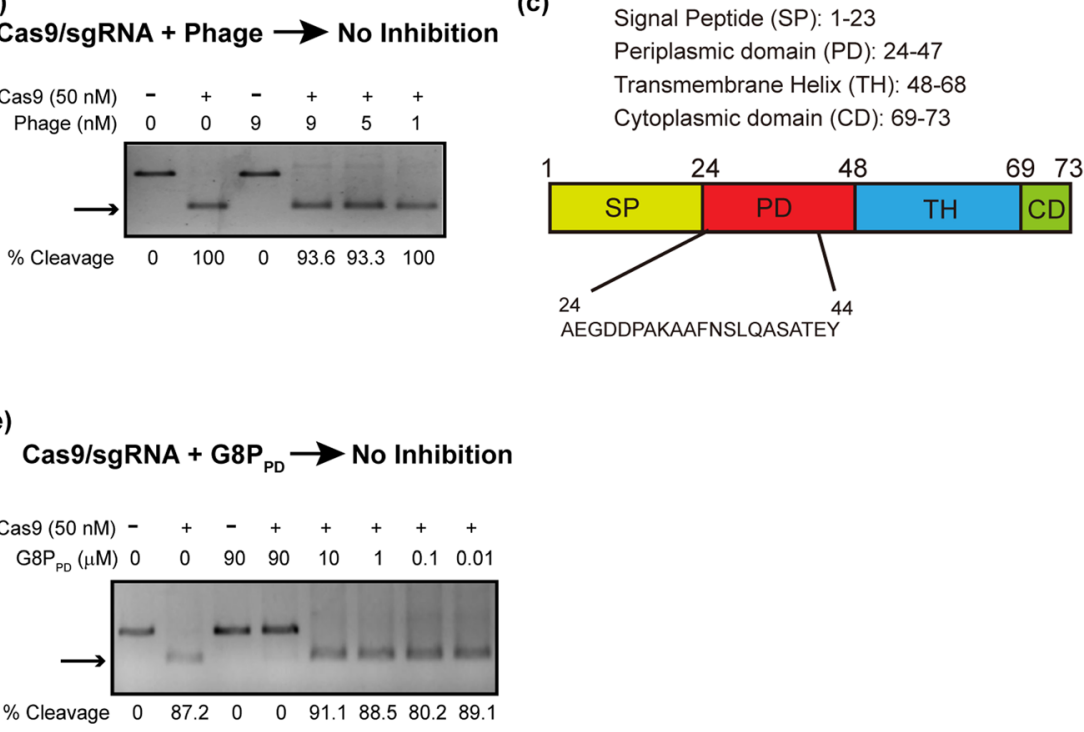

Fig. 1 Inhibition of the in vitro activity of SpCas9 by intact M13 phage and phage-derived G8P PD peptides. a Dose-dependent inhibition of SpCas9 by intact M13 phage. b Intact M13 phage does not inhibit the in vitro activity of assembled Cas9-sgRNA RNP. c Structural organization of M13 phage major coat protein G8P. $\mathbf{d}$ Dose-dependent inhibition of SpCas9 by G8P PD. e G8P PD does not inhibit the in vitro activity of assembled Cas9-sgRNA RNP. The above reactions are performed in the absence or presence of $50 \mathrm{nM}$ SpCas9 proteins. The results are shown as mean \pm SD $(n=3)$. Arrows indicate cleavage products

(see below for detailed characterization). Interestingly, G8P $P_{P D}$ peptides from $\mathrm{f} 1$ and M13 phages have only one amino acid difference (Additional file 1: Figure S1a) and similar activities on inhibiting Cas9 cleavage or Cas9sgRNA binding (Additional file 1: Figure S1b-c). The synthetic f1 G8P $\mathrm{PD}_{\mathrm{PD}}$ oligopeptide seemed to have higher aqueous solubility and is used interchangeably with M13 $\mathrm{G}_{\mathrm{P}} \mathrm{PD}_{\mathrm{PD}}$ in the following studies.

\section{G8P $_{\text {PD }}$ prevents the assembly of Cas9 and sgRNA by binding to sgRNA-free Cas9 (apo-Cas9)}

SpCas9 can be inactivated at distinct steps during its action including guide RNA binding, substrate DNA binding and DNA cleavage. Most previously known Acrs exert their inhibitory activity by interfering with DNA surveillance or cleavage. To understand the mechanism of actions of $\mathrm{G}_{\mathrm{PD}}$, we examined the interactions between G8P $_{\mathrm{PD}}$ and apo-Cas9 or Cas9-sgRNA RNP using electrophoresis mobility shift assay (EMSA). Under fixed sgRNA concentration of $15 \mu \mathrm{M}$, the fraction of Cas9bound sgRNA increased with the increasing molar ratio of Cas9 and sgRNA in the absence of G8P $\mathrm{PD}_{\mathrm{PD}}$. By contrast, pre-incubation of SpCas 9 proteins with $300 \mu \mathrm{M}$ G8P $P_{\mathrm{PD}}$ reduced the fraction of Cas9-bound sgRNA in a G8P $P_{\text {PD }}$ dose-dependent manner (Fig. 2a). We also noticed that under high molar ratio of Cas9 and sgRNA, $\mathrm{G}_{\mathrm{PD}}$ did not fully block the formation of Cas9-sgRNA RNP (Fig. 2a), suggesting a weak interaction between
Cas9 and $\mathrm{G}_{\mathrm{PD}}$ that is reversible under high concentrations of sgRNA.

$\mathrm{G}_{\mathrm{PP}} \mathrm{P}_{\mathrm{PD}}$-mediated inhibition of Cas9-sgRNA assembly is dependent on the order of sgRNA addition. When the ratio of the molar concentrations of Cas 9 and sgRNA is fixed to 1:0.3, pre-incubation of Cas9 with 300 or $600 \mu \mathrm{M}$ f1 $\mathrm{G} \mathrm{P}_{\mathrm{PD}}$ prior to sgRNA addition abolished the assembly of Cas9 and sgRNA (Fig. 2b). By contrast, supplementation of 300 or $600 \mu \mathrm{M}$ G8P $\mathrm{PD}_{\mathrm{PD}}$ post sgRNA addition had minor or no effect on the formation of Cas9-sgRNA complex (Fig. 2b). These results suggest that $\mathrm{G}_{\mathrm{PD}}$ prevents Cas9-sgRNA binding by interacting with apo-Cas9 but not sgRNA-bound Cas9 and may explain why $\mathrm{G}_{8} \mathrm{P}_{\mathrm{PD}^{-}}$-mediated inactivation of SpCas9 cleavage is dependent on the order of sgRNA addition.

\section{Identification of $\mathrm{G}_{\mathrm{PPD}}$ binding site in SpCas9}

In order to dissect the mechanism of interactions between SpCas9 and $\mathrm{G}_{\mathrm{PP}}$, we sought to determine the binding region of $\mathrm{G}_{\mathrm{P}} \mathrm{P}_{\mathrm{PD}}$ on SpCas9 using highresolution mass spectrometry (MS). SpCas9 protein and M13 G8P $_{\mathrm{PD}}$ were crosslinked using collision-induced dissociation (CID)-cleavable crosslinker disuccinimido sulfoxide (DSSO) [55] and then were subject to digestion with chymotrypsin. The integration analyses of CIDinduced cleavage of interlinked peptides in MS/MS and $\mathrm{MS}^{3}$ of single peptide chain fragment ions revealed high crosslinking scores (Fig. 3a) on residues K1158 of [K] 


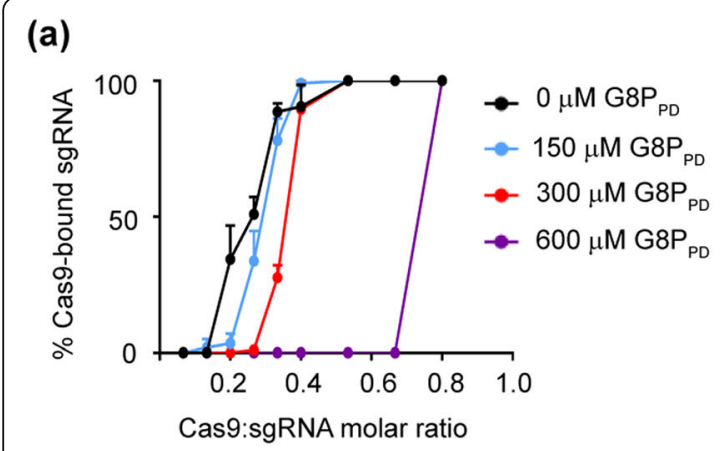

(b)
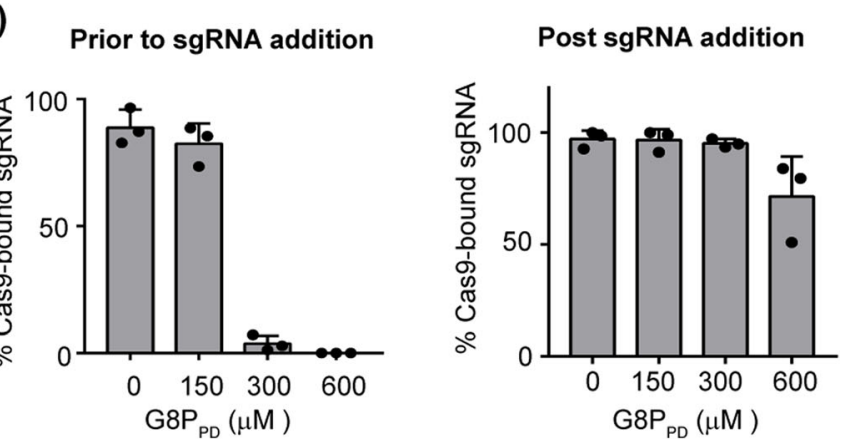

Fig. 2 G8P PD prevents Cas9-sgRNA assembly. a Dose-dependent inhibition of Cas9-sgRNA binding by f1 G8P PD. sgRNA concentration is fixed to $15 \mu \mathrm{M}$. b f1 G8P PD prevents Cas9-sgRNA assembly prior to, but not post, sgRNA addition. Cas9 to sgRNA ratio is fixed to 0.3. The above results are shown as mean $\pm \mathrm{SD}(n=3)$

SVKEL peptide and K1176 of E [K] NPIDFLEAKGY peptide from SpCas9 (Fig. 3b, c and Additional file 1: Figure S2a-b). These peptides occupy a continuous region in the PAM-interacting (PI) domain of SpCas9 (Fig. 3d) that is responsible for recognizing the PAM sequence on the non-complementary DNA strand [56]. Interestingly, this candidate $\mathrm{G}_{\mathrm{P}} \mathrm{P}_{\mathrm{PD}}$ binding site dose not locate in the sgRNA or DNA binding pockets of SpCas9. These results suggest that $G 8 \mathrm{P}_{\mathrm{PD}}$ does not directly compete with sgRNA but may instead function as an allosteric inhibitor.

Next we sought to perform mutational analyses on the candidate $\mathrm{G}_{\mathrm{PD}} \mathrm{P}_{\mathrm{PD}}$ binding sites in SpCas9. Residues KSKVEL in K1158 mutant and EKNPID in K1176 mutant were mutated into alanines respectively. Mutant SpCas9 proteins were purified into high homogeneity (Additional file 1: Figure S3a-b). In vitro cleavage reaction illustrated that alanine mutations at positions

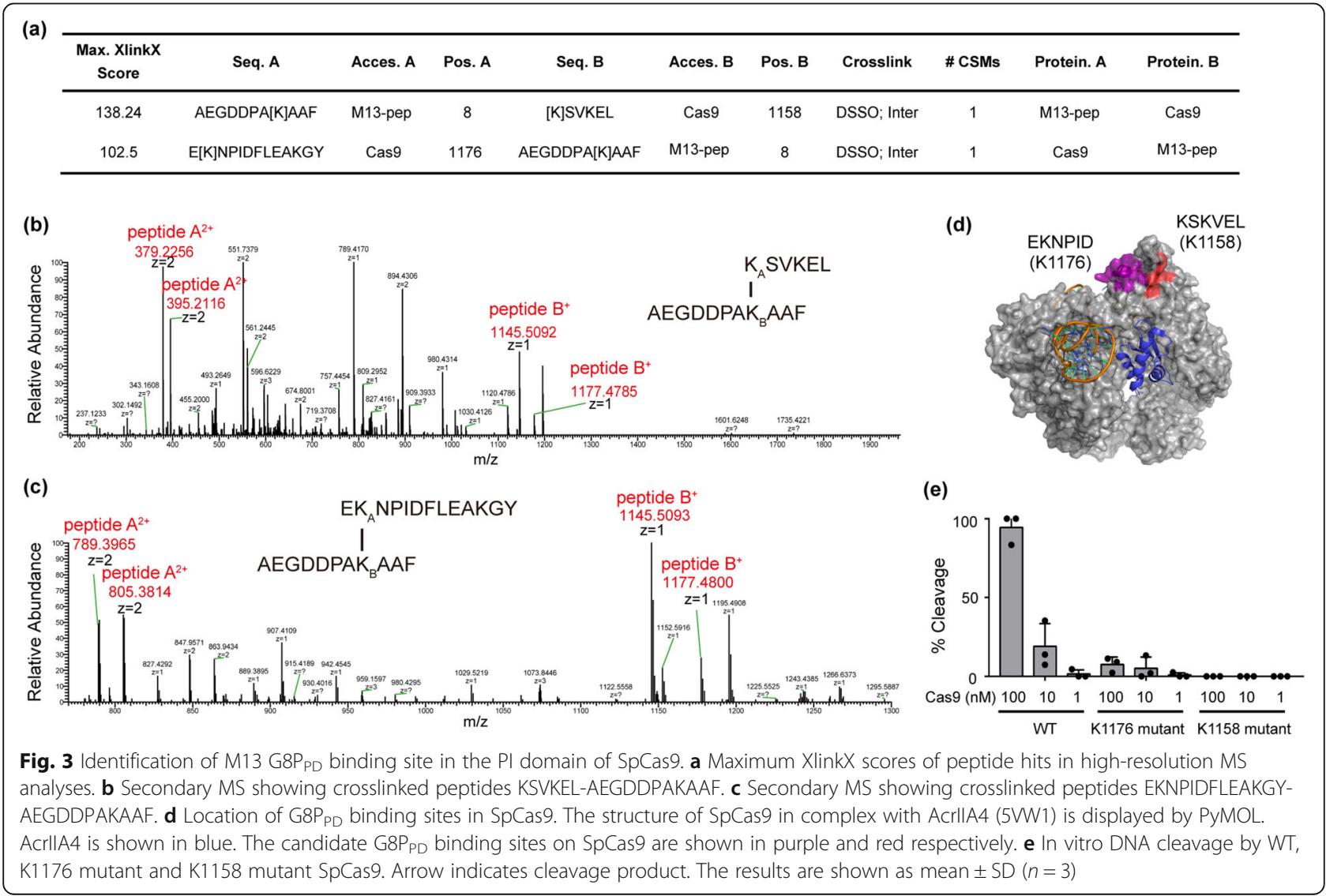


KSKVEL (K1158 mutant) and EKNPID (K1176 mutant) markedly reduced the DNA cleavage activity of SpCas9 (Fig. 3e), suggesting the importance of the G8P $\mathrm{PD}_{\mathrm{PD}}$ binding sites for SpCas9 activity.

\section{a-Helical structure is critical for the inhibitory activity of G8P $P_{P D}$}

Next we performed an alanine scan on $\mathrm{f} 1$ phage $\mathrm{G} 8 \mathrm{P}_{\mathrm{PD}}$ to illustrate its structural determinants for Cas9 inhibition. Four peptide mutants are designed with alanine mutations spanning the entire G8 $\mathrm{P}_{\mathrm{PD}}$ sequence (Fig. 4a). Alanine mutations at positions 6 to 11 abolished the inhibitory activity of $\mathrm{G}_{8} \mathrm{P}_{\mathrm{PD}}$ while mutants 1,3 , and 4 retained the majority of Cas9-inhibiting activity (Fig. 4a). The major coat protein G8P adopts an $\alpha$-helical structure [54]. Residues 6 to 11 are located at the $\mathrm{N}$-terminus of the $\alpha$ helix and contain Pro6, Lys8, Phe11, and three native alanines (Fig. 4b). The abolished activity in mutant 2 suggested a critical role of positions 6 to 11, particularly the residues Pro6, Lys8, and Phe11. Circular dichroism (CD) spectra study revealed an $\alpha$-helical structure-enriched feature for $\mathrm{f1}$ G8 $\mathrm{P}_{\mathrm{PD}}$ WT but not for mutant 2 (Fig. 4c), suggesting that Pro6, Lys8, and Phe11 are important for maintaining the $\alpha$-helical structure in $\mathrm{f} 1 \mathrm{G} 8 \mathrm{P}_{\mathrm{PD}}$. Although the presence of residues 6 to 11 could directly participate in the interaction between Cas 9 and G8P $\mathrm{PD}_{\mathrm{PD}}$, the overall $\alpha$ - helical structure of G8P $\mathrm{P}_{\mathrm{PD}}$ could be also important for its Cas9-inhibiting activity.

\section{Modulation of SpCas9 activity in human cells using inoviridae phage $\mathrm{G}_{\mathrm{PPD}}$}

We next explored the potential application of G8P $\mathrm{P}_{\mathrm{PD}}$ as an off switch for the genome-editing activity of SpCas9 in human cells. Ectopic expression of the full-length $\left(\mathrm{G}_{\mathrm{FL}}\right)$ or periplasmic domain $\left(\mathrm{G} 8 \mathrm{P}_{\mathrm{PD}}\right)$ of $\mathrm{M} 13$ and $\mathrm{f} 1$ G8Ps at $24 \mathrm{~h}$ prior to Cas9-sgRNA transfection significantly suppressed the genome-editing activity of SpCas9 in HEK293 cells (Fig. 5a, b). In comparison, Acr protein AcrII4A [38] blocked SpCas9 cleavage on AAVS1 site whereas Neisseria meningitidis Cas9 (NmeCas9)-specific Acr protein AcrIIC3 [57] partially inhibited SpCas9. Importantly, f1 G8P $P_{P D}$ was capable to inhibit SpCas9 activity across different genes and cell types (Fig. 5b, c). Consistent with the in vitro experiments, significant inhibition of the on-target activity of SpCas9 in human cells was observed only when $\mathrm{G}_{\mathrm{P}} \mathrm{P}_{\mathrm{PD}}$ was overexpressed prior to sgRNA transfection. Co-transfection of $\mathrm{G}_{\mathrm{P}} \mathrm{P}_{\mathrm{PD}}$ and SpCas9-sgRNA did not inhibit SpCas9 cleavage $(P>0.05)$ (Fig. 5d).

In order to have detailed understanding of the effects of G8P $\mathrm{PD}_{\mathrm{PD}}$ on the genome-editing activity of SpCas9, we performed next-generation sequencing (NGS) to analyze the profiles of edited genomic loci in the absence and

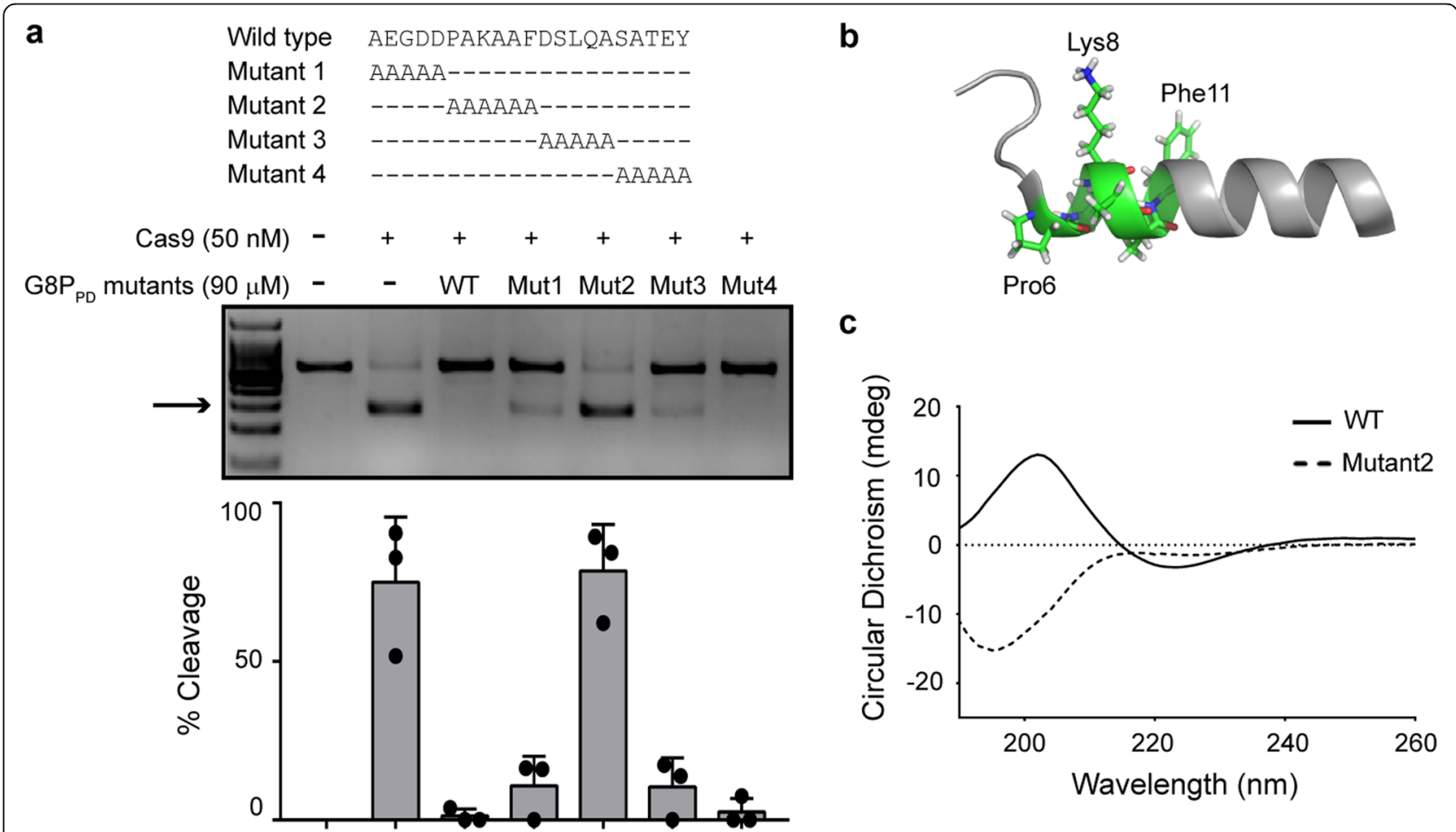

Fig. 4 a-helical structure is critical for the Cas9-inhibiting activity of G8P PD. a Alanine mutations at positions 6-11 abolish the inhibitory activity of $\mathrm{G}_{\mathrm{PPD}}$. The results are shown as mean $\pm \mathrm{SD}(n=3)$. The arrow denotes cleavage products. $\mathbf{b}$ Structure of G8P PD peptide (PDB entry 2MJZ), displayed by PyMOL. Residues 6-11 are shown as stick. c CD spectra of f1 G8P $\mathrm{PD}$ WT and mutant 2 


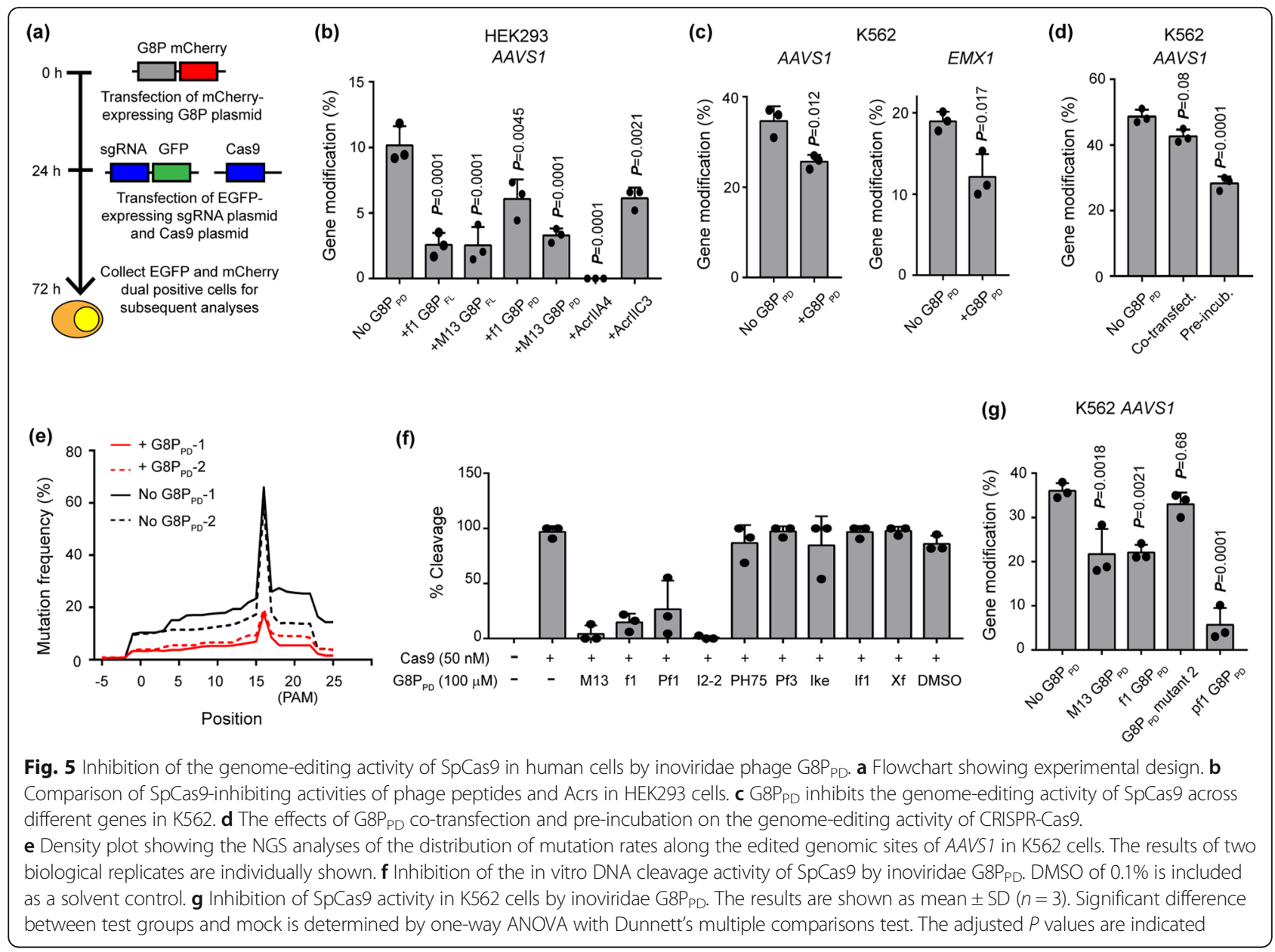

presence of $\mathrm{G}_{8 \mathrm{PD}}$. Despite a reduced mutation rate, the mutation pattern of SpCas9 along the 20-bp sgRNAtargeting site was not altered by $\mathrm{G}_{\mathrm{P}} \mathrm{P}_{\mathrm{PD}}$ treatment, as characterized by the high-frequency editing events at $3 \mathrm{bp}$ upstream of the PAM sequence [4] (Fig. 5e). Importantly, G8P $P_{P D}$ treatment retained the distribution pattern of indel length, with $1-5 \mathrm{bp}$ indel being predominant in the population (Additional file 1: Figure S4a). In addition, we observed modest decrease in the in-frame mutations $(3 \mathrm{~N})$ (Additional file 1: Figure S4b), the mechanism of which is yet to be elucidated. Collectively, these data suggested that $\mathrm{G}_{\mathrm{PP}} \mathrm{P}_{\mathrm{PD}}$ treatment did not cause major alterations in the profiles of SpCas9-induced mutations, thus highlighting the potential of $\mathrm{G}_{\mathrm{P}} \mathrm{PD}_{\mathrm{PD}}$ as a safe off switch for the therapeutic applications of SpCas9.

To expand peptide-based anti-CRISPR toolbox, we examined the G8Ps from other inoviridae phages (Additional file 1: Figure S5). Peptides constituting the periplasmic domain of these G8P ( $\left.68 \mathrm{P}_{\mathrm{PD}}\right)$ are synthesized and evaluated for the in vitro and in vivo activities. At a concentration of $100 \mu \mathrm{M}$, the G8P $\mathrm{PD}_{\mathrm{PD}}$ from M13, f1, Pf1, and I2-2 phage markedly inhibited the in vitro DNA cleavage activity of SpCas9 while other $\mathrm{G} 8 \mathrm{P}_{\mathrm{PD}}$ orthologues showed little inhibitory effects (Fig. 5f). Ectopic expression of M13, f1, and pf1 G8P $\mathrm{PD}_{\mathrm{PD}}$ in $\mathrm{K} 562$ cells significantly reduced the activity of SpCas9 whereas G8P $\mathrm{PD}_{\mathrm{PD}}$ mutant 2 did not show inhibitory activity $(P>0.05)$ (Fig. $5 \mathrm{~g})$. Our results suggested that inoviridae phage G8Ps could be leveraged to inhibit both the in vitro and in vivo activity of SpCas9. The variations of $\mathrm{G}_{\mathrm{PD}}$ sequences and the difference in their inhibitory activities indicate that further engineering endeavors could be made to improve Cas9-inhibiting peptides.

To understand the specificity of $\mathrm{G} \mathrm{P}_{\mathrm{PD}}$ within type II CRISPR system, we examine the inhibitory activity of $\mathrm{f} 1$ G8P $\mathrm{P}_{\mathrm{PD}}$ on Cas9 orthologues from Staphylococcus aureus (SaCas9) and Neisseria meningitidis (NmCas9). NGS analyses showed that pre-incubation of HEK293 cells with $\mathrm{G} \mathrm{P}_{\mathrm{PD}}$ did not significantly inhibit the activities of NmCas9 or SaCas9 at the examined genomic sites (Additional file 1: Figure S6).

\section{G8P co-transfection improves the specificity of SpCas9 in human cells}

It has been reported that timed delivery of AcrIIA4 can improve the genome-editing specificity of nucleofected Cas9-sgRNA RNP complex [38]. We intended to 
investigate the effects of G8P peptides on the specificity of constitutively expressed SpCas9, a more therapeutically relevant model. The aforementioned in vitro and in vivo data have suggested that the inhibitory effects of G8P on CRISPR-Cas9 is dependent on the accessibility of Cas9 protein to sgRNA. This observation prompted us to explore whether G8P can be leveraged, via timed delivery, to improve the specificity of SpCas9. Unlike the above experiments using G8Ps as CRISPR-Cas9 off switch (Fig. 5), here we co-transfected Hela cells with Acr or G8P plasmid and sgRNA and SpCas9-coding plasmids. AcrIIA4 suppressed the on- and off-activities of SpCas9 to undetectable levels, as determined by T7E1 assay (Fig. 6a). NGS analyses showed that M13 G8P $\mathrm{FL}_{\mathrm{FL}}$ and f1 G8P $\mathrm{PL}_{\mathrm{FL}}$ significantly reduced $(P<0.05)$ both ontarget and off-target activity of SpCas9 in Hela cells. It appeared that the inhibitory effects are more prominent at the off-target site (Fig. 6b). AcrIIC3 significantly reduced the on-target activity $(P<0.05)$ but not the offtarget activity $(P>0.05)$ (Fig. 6b). Importantly, M13
G8P $P_{P D}$ could reduce the off-target events without affecting the on-target cleavage (Fig. 6a, b).

Similarly, M13 G8P $\mathrm{PD}_{\mathrm{PD}}$ reduced the off-target events of SpCas9 in K562 cells but retained the on-target activity (Fig. 6c). Co-transfection of M13 G8P $\mathrm{PL}_{\mathrm{FL}}$ or $\mathrm{f} 1 \mathrm{G} \mathrm{P}_{\mathrm{FL}}$ in K562 cells led to minimum or no significant decrease of the on-target activity of SpCas9 but markedly reduced the off-target events (Fig. 6c). Surprisingly, in K562 cells, AcrIIC3 had little effect on the on-target activity of SpCas9 but notably increase the off-target activity (Fig. 6c).

Examination of the effects of co-transfected G8Ps on SpCas9 at another therapeutically relevant genomic site $H B B$ [58] illuminated that G8Ps could significantly reduce off-target activity with little perturbation of the on-target activity (Additional file 1: Figure S7). Moreover, G8P treatment did not alter the patterns of CRISPR-induced mutations, as characterized the positions of peak mutations (Additional file 1: Figure S7). Collectively, these data are in line with the observation at AAVS1 site and suggest that co-transfection of G8P-based

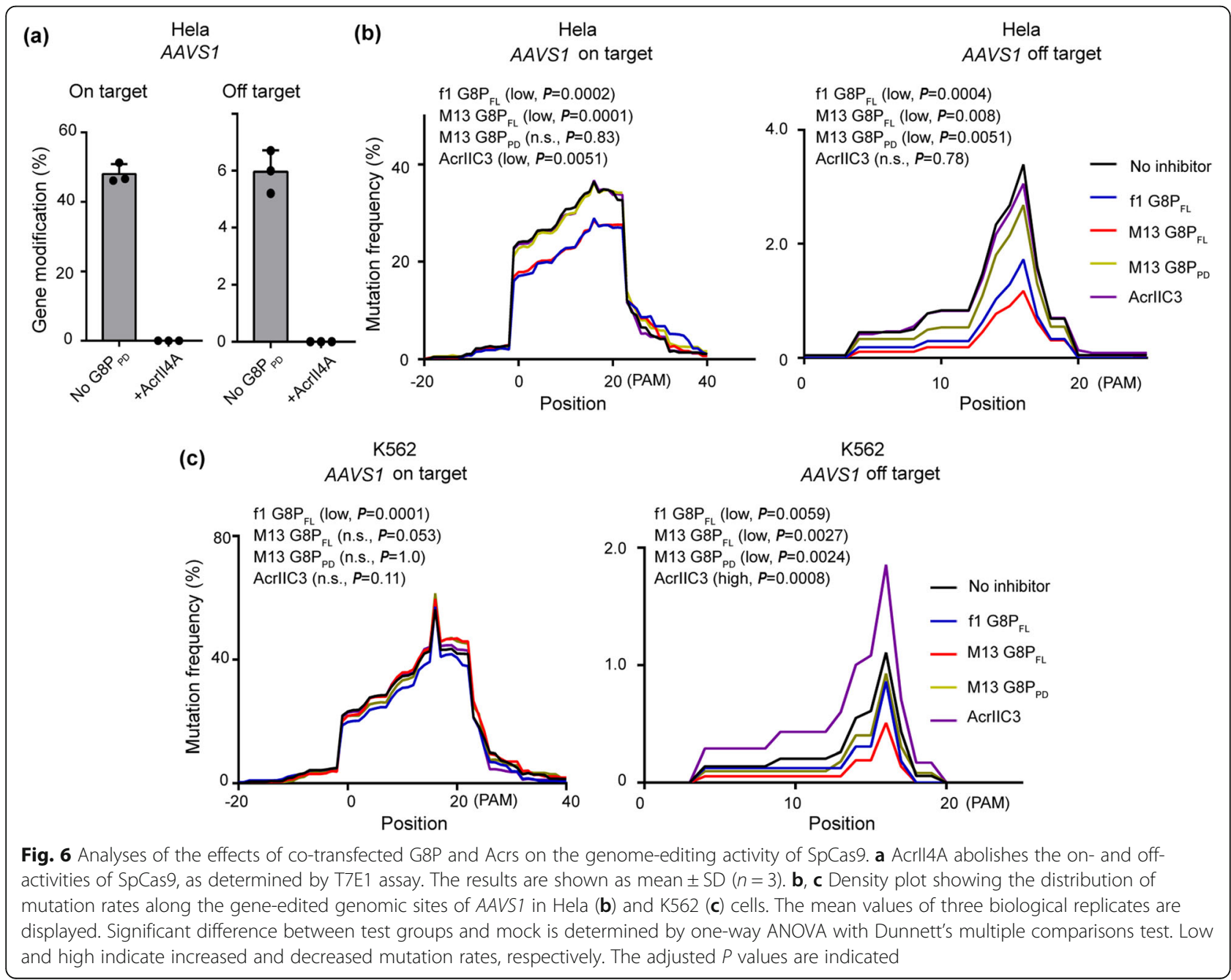


anti-CRISPR agents can reduce the off-target events of constitutively expressed SpCas9 in human cells with minimum perturbation on the on-target activity.

CCK-8 assay revealed little or no change of the cell viability of HEK293 cells that were pre-incubated or cotransfected with CRISPR inhibitors (Additional file 1: Figure S8), ruling out the possibility that the observed inhibitory effects of G8Ps are the results of associated cytotoxicity. To explore the possible mechanism of G8Pmediated improvement of CRISPR-Cas9 targeting, we performed chromatin immunoprecipitation-quantitative PCR (ChIP-qPCR) to analyze the effects of G8P on the binding of a catalytically inactive Cas9 (dCas9) at the AAVS1 on target and pre-determined off-target sites. It was found that the presence of M13 G8P $\mathrm{PD}_{\mathrm{PD}}$ or M13 G8P $\mathrm{P}_{\mathrm{FL}}$ did not significantly improve the binding specificity at on-target site (Additional file 1: Figure S9). This suggested that G8P-mediated improvement of CRISPRCas9 targeting was unlikely attributed to improved Cas9 binding. Further studies are required to illustrate the detailed mechanism of action of G8P in living cells.

\section{Discussion}

In this study, we reported the discovery of anti-CRISPR peptides from inoviridae bacteriophages. The clue leading to this discovery was based on the observation that intact M13 phage inhibited the DNA cleavage activity of SpCas9 in in vitro reactions. Although we focused the investigation on the major coat protein G8P in the present study, it is likely that other surface-exposed minor coat proteins may attribute to intact phagemediated SpCas9 inhibition.

Unlike previously described Acrs that inhibit CRISPRCas by disrupting DNA binding $[36,45,59]$ or DNA cleavage [36], G8P inactivates CRISPR-Cas9 via a distinct mechanism by preventing Cas9 from sgRNA binding. Moreover, to achieve efficient in vitro and in vivo inhibition, G8 $\mathrm{P}_{\mathrm{PD}}$ must access SpCas9 prior to its binding with sgRNA, indicating that G8 $\mathrm{P}_{\mathrm{PD}}$ binds to apo-Cas9, but not sgRNA-bound Cas9. These results suggest that G8P $P_{P D}$ and sgRNA are mutually exclusive for binding with Cas9 nuclease. One straightforward explanation is that $\mathrm{G} 8 \mathrm{P}_{\mathrm{PD}}$ and sgRNA compete for the same binding pocket. However, MS and mutational studies suggest that the binding site of $\mathrm{f1} \mathrm{G}_{\mathrm{P}} \mathrm{PD}_{\mathrm{PD}}$ is located on the PI domain of Cas9, distal from sgRNA or DNA binding pockets, thus suggesting against direct competition between G8P $\mathrm{PD}_{\mathrm{PD}}$ and sgRNA for the same binding pocket. These results strongly suggest that $\mathrm{GPP}_{\mathrm{PD}}$ function as allosteric inhibitors to SpCas9.

As allosteric inhibitors, G8P $\mathrm{PD}_{\mathrm{PD}}$ may prevent Cas9sgRNA binding by introducing steric hindrance or conformational changes. It is known that the binding of guide RNA can induce conformational rearrangements of Cas9 [60]; thus, it is possible that $\mathrm{G} \mathrm{P}_{\mathrm{PD}}$ or sgRNA binding can transform SpCas9 from a flexible conformation to a closed conformation, which prevents SpCas9 from binding with the other counterpart. Additional experiments are required to better illustrate the mechanism of SpCas9 inhibition by G8P. Collectively, G8Pmediated allosteric inhibition of Cas9 and sgRNA binding represents a unique CRISPR-inactivating mechanism that may have important implications for developing next-generation anti-CRISPR agents.

Existing Acr proteins can display nanomolar binding affinity to Cas9 [36]. By contrast, inoviridae phage G8P $_{\mathrm{PD}}$ peptides exhibit weak affinity as evidenced by the micromolar $\mathrm{IC}_{50}$ in the in vitro cleavage reaction (Fig. 1) and by its inability to completely block the assembly of Cas9 and sgRNA (Fig. 2). Interestingly, intact M13 phage inhibits Cas 9 with an $\mathrm{IC}_{50}$ of approximately $5 \mathrm{nM}, 1000$ fold lower than that with $68 \mathrm{P}_{\mathrm{PD}}$. The increased potency with intact phage may result from the enhanced cooperativity and avidity afforded by the multimeric assembly of the phage capsid that carries 2700 copies of G8P.

Although we have demonstrated that inoviridae phage G8P $P_{P D}$ can function as anti-CRISPR peptides, the biological relevance of our discovery is yet to be explored. Under native context, the major coat protein G8P does not enter bacterial cytoplasm during phage infection. It is thus unlikely for G8P to exert anti-CRISPR function at the early stage of phage infection. Instead, G8P could interact with CRISPR-Cas in bacterial cytoplasm after the phage genome is translated. This post-translational inhibitory activity will require G8P-coding DNA to evade CRISPR attack during phage infection. In addition, the SpCas9inactivating $\mathrm{G} \mathrm{P}_{\mathrm{PD}}$ discovered in the present study are encoded by phages that infect Escherichia coli and Pseudomonas aeruginosa, which do not harbor type II CRISPRCas system. Horizontal gene transfer could explain for the cross-species CRISPR inactivation [26, 32]; however, a systemic phylogenetic analysis is necessary to reveal the evolutionary implications of the anti-CRISPR activity of G8Ps.

Despite the elusive biological relevance of the antiCRISPR activity of G8P, we nevertheless demonstrated that the genome-editing activity of SpCas9 in human cells can be modulated by G8P $\mathrm{PD}_{\mathrm{PD}}$. To the best of our knowledge, our discovery represents the first peptides known to exhibit Cas9-inhibiting activity and expands the anti-CRISPR agent toolbox which is currently composed of anti-CRISPR proteins [21], small molecules [20], and synthetic oligonucleotides [61]. Compared with Acr proteins that are typically 10 to $20 \mathrm{kD}$ in size, G8P $_{P D}$ peptides are small in size and can be chemically synthesized in a large scale. The facile manufacturing process is critical for the rapid evaluation of the structure-activity relationship to identify enhanced anti-CRISPR peptides. The varied Cas9-inhibiting activities observed among different inoviridae phage 
G8P $P_{P D}$ supports the notion of improving SpCas9inhibiting peptides by sequence optimization. In addition, the differential effects of G8Ps on SpCas9, NmCas9, and SaCas9 indicate that it may be feasible to design specific peptide inhibitors for different Cas9 homologs.

G8P $\mathrm{P}_{\mathrm{PD}}$ does not alter the pattern of SpCas9-induced mutations, suggesting that $\mathrm{G}_{8} \mathrm{P}_{\mathrm{PD}}$-mediated Cas9 inactivation does not interfere with the downstream DNA repair pathway. This feature facilitates the therapeutic applications of G8P $\mathrm{PD}_{\mathrm{PD}}$ as CRISPR-Cas off switches by restricting the alterations of genome-editing outcome. It has been demonstrated that increasing the intracellular concentrations of Cas9 can yield elevated off-target activity without further increasing the saturated on-target cleavage, thereby resulting in decreased specificity of genome targeting [12]. It has been proposed that the specificity of SpCas9 in human cells may be increased by partial inhibition with weak CRISPR-Cas inhibitors [20]. In the current study, we found that transfection of strong CRISPR-Cas inhibitor AcrII4A inhibited SpCas9 activity to near complete in human cells when transfected prior to (Fig. 5b) or simultaneously with (Fig. 6a) sgRNA- and SpCas9-coding plasmids. By contrast, cotransfection of G8P with CRISPR-Cas9 showed little inhibition of the on-target activity but reduced the offtarget events at endogenous genomic sites (Fig. 6 and Additional file 1: Figure S7).
Therefore, we propose a two-mode mechanism of action for G8P to suppress SpCas9 activities in living cells: pre-incubation of G8P can saturate the intracellular concentrations of G8P and efficiently inhibit the activity of subsequently transfected Cas9 by competing with sgRNA for binding with apo-Cas9, while co-transfection of G8P with Cas9 and sgRNA will not affect the assembly of Cas9-sgRNA for cleavage at on-target sites but only prevent excess Cas9 from off-target editing (Fig. 7). Interestingly, it was observed that the AcrIIC3, a weak inhibitor to SpCas9 (Fig. 5b), did not increase the specificity of SpCas9 in human cells (Fig. 6b, c). This result along with the results of $\mathrm{G} 8 \mathrm{P}_{\mathrm{PD}}$ suggests that the mechanism of action of the inhibitors may be also important for their effects on the specificity of CRISPR-Cas9. In addition, it has to be noted, however, that the applicability of G8Ps as agents to improve the specificity of CRISPR-Cas9 requires further investigation on additional cell types, genomic loci, and genome-wide mutation profile and improved understanding of the mechanism of action of G8P.

\section{Conclusion}

In the present study, we report the surprising discovery of phage-derived peptides that can inhibit the in vitro and in vivo activities of SpCas9. These peptides inhibited Cas 9 activity by disrupting Cas 9 and sgRNA binding in an allosteric manner. We show that the genome-editing

G8P Pre-incubation

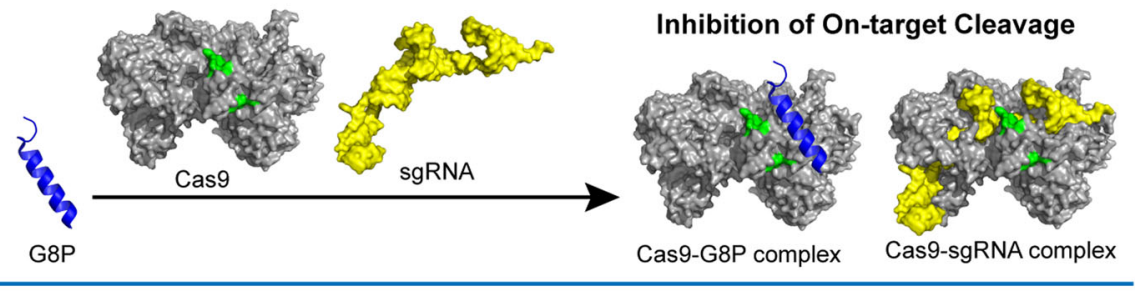

G8P Co-transfection

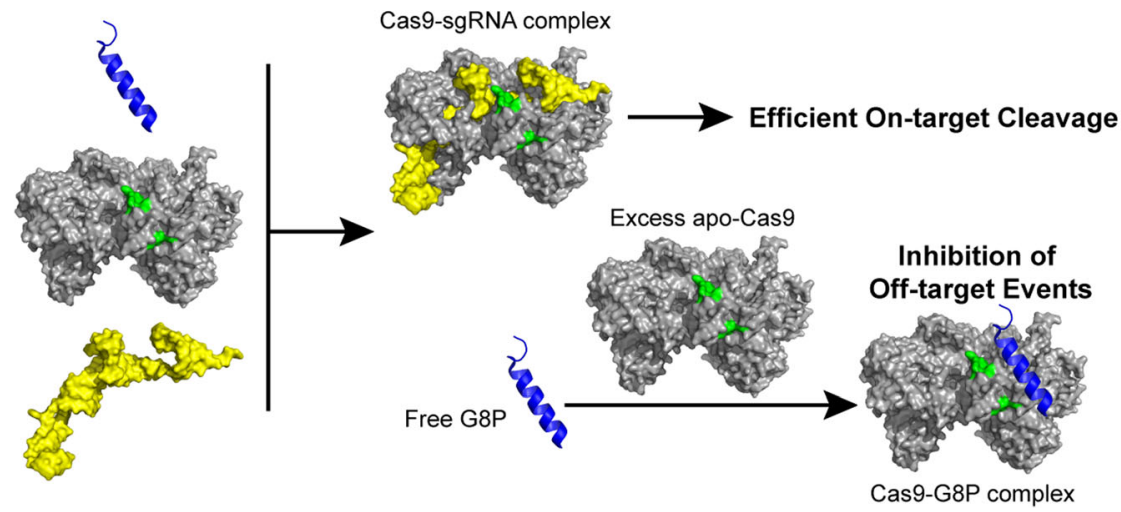

Fig. 7 Proposed mechanism of action of G8P in living cells when delivered prior to or simultaneously with Cas9 and sgRNA-coding genes 
activity of CRISPR-Cas9 can be harnessed by G8P. This unique mechanism of action of G8P may provide insights into developing anti-CRISPR agents towards genome- or base-editing systems.

\section{Methods}

\section{Production of M13 bacteriophage}

To produce M13 phage, XL1-Blue E. coli was inoculated in SB medium supplemented with $2 \%$ glucose and grown until $\mathrm{OD}_{600}$ reached 0.5 . M13 phages were transfected and incubated with XL1-Blue E. coli at $37^{\circ} \mathrm{C}$ for $30 \mathrm{~min}$ and the culture was shaken at $37^{\circ} \mathrm{C}$ for $1 \mathrm{~h}$. Thereafter, bacteria were centrifuged twice at $3000 \mathrm{rpm}$ for $15 \mathrm{~min}$ at $4{ }^{\circ} \mathrm{C}$ and washed with $\mathrm{SB}$ medium to remove glucose and free phage particles. The cells were re-suspended with SB medium and diluted to an $\mathrm{OD}_{600}$ of 0.8 in 200 $\mathrm{mL}$ culture and grown at $30{ }^{\circ} \mathrm{C}$ overnight with shaking. The next day, the culture was centrifuged at $5000 \mathrm{rpm}$ for $15 \mathrm{~min}$ at $4{ }^{\circ} \mathrm{C}$ and the supernatant containing phage particles was collected. The supernatant was gently mixed with NaCl-PEG buffer containing 20\% PEG and $2.5 \mathrm{M} \mathrm{NaCl}$ and kept on ice for $1 \mathrm{~h}$. The PEG-phage supernatant was centrifuged at $9000 \mathrm{rpm}$ for $30 \mathrm{~min}$ at $4{ }^{\circ} \mathrm{C}$. The supernatant was discarded and phage pellet was centrifuged again at $9000 \mathrm{rpm}$ for $5 \mathrm{~min}$ at $4{ }^{\circ} \mathrm{C}$ to remove residual medium. The precipitated phage particle was re-suspended in phosphate buffered saline (PBS) for further studies.

\section{Cell culture}

HEK293T cells were maintained in Dulbecco's modified Eagle's medium (DMEM; Gibco/Thermo Fisher Scientific, Shanghai, China) supplemented with $10 \%$ fetal bovine serum (FBS, Gibco/Thermo Fisher Scientific) at 5\% $\mathrm{CO}_{2}$ and $37^{\circ} \mathrm{C}$ in a fully humidified incubator and were passaged when $70-90 \%$ confluency was reached. K562 cells were cultured in RPMI-1640 medium supplemented with $10 \% \mathrm{FBS}, 100 \mathrm{IU} / \mathrm{mL}$ of penicillin, and $100 \mu \mathrm{g} / \mathrm{mL}$ of streptomycin at $37^{\circ} \mathrm{C}$ under $5 \% \mathrm{CO}_{2}$. Cell lines were validated by VivaCell Biosciences (Shanghai, China). Cell viability was determined using CCK-8 assay (Sigma-Aldrich, Shanghai, China).

\section{Expression and purification of SpCas9 proteins}

pET28b plasmids coding SpCas9 WT, K1158 mutant and K1176 mutant proteins were transformed into $E$. coli BL21 (DE3) cells. Single colonies were picked and grown in 2-L LB media supplemented with $50 \mu \mathrm{g} / \mathrm{mL}$ kanamycin. Culture was grown to an $\mathrm{OD}_{600}$ of 0.8 . Protein expression was induced with $0.2 \mathrm{mM}$ isopropyl- $\beta$-Dthiogalactopyranoside (IPTG) at $16^{\circ} \mathrm{C}$ overnight. Cells from 2-L culture were pelleted by centrifugation at $6000 \mathrm{~g}$ at $4{ }^{\circ} \mathrm{C}$ for $15 \mathrm{~min}$ and then re-suspended in 40 $\mathrm{mL}$ binding buffer containing $20 \mathrm{mM}$ Tris $\mathrm{HCl}, \mathrm{pH} 8.0$ and $0.5 \mathrm{M} \mathrm{NaCl}$. Cell suspension was then supplemented with $1 \mathrm{mM}$ Tris (2-carboxyethyl) phosphine (TCEP) and $1 \times$ complete inhibitor cocktail (Roche). Cells were lysed by sonication on ice and then centrifuged at $80,000 \mathrm{~g}$ at $4{ }^{\circ} \mathrm{C}$ for $30 \mathrm{~min}$. The supernatant of cell lysate was incubated with $1 \mathrm{~mL} \mathrm{Ni-NTA}$ agarose beads (QIAGEN) at $4{ }^{\circ} \mathrm{C}$ for $1 \mathrm{~h}$. The resin was washed with $20 \mathrm{~mL}$ wash buffer that was made by supplementing binding buffer with $30 \mathrm{mM}$ imidazole. Proteins were eluted with $5 \mathrm{~mL}$ elute buffer that was made by supplementing binding buffer with $300 \mathrm{mM}$ imidazole. Eluted protein samples were further purified by gel filtration using Superose 6 10/300 column (GE Healthcare). These proteins were bufferexchanged to storage buffer containing $20 \mathrm{mM}$ HEPES, pH 8.0 and $200 \mathrm{mM} \mathrm{NaCl}$, aliquoted and stored at $-80^{\circ} \mathrm{C}$.

\section{Construction of $\mathrm{G}_{8 \mathrm{PD}}$ overexpression plasmids}

Human codon-optimized DNA sequences encoding M13, f1, f1 mutant 2, and pf1 G8P $\mathrm{P}_{\mathrm{PD}}$ were cloned into the BamHI/XbarI sites of pcDNA3.1(+) by plasmid recombination kit Clone Express (Vazyme). These G8P $P_{P D}$ peptides carry an N-terminal SV40 nuclear localization signal (NLS) for co-localization with Cas9 proteins. G8 $P_{P D}$ peptides were cloned into plv-EF1 $\alpha$-mCherry plasmid harboring mCherry fluorescent protein marker. sgRNA was cloned into pGL3-U6-gRNA plasmid carrying green fluorescent protein (GFP).

\section{In vitro transcription of sgRNA}

CCR5-targeting sgRNA (Additional file 2: Table S1) was transcribed from a sgRNA-coding PCR product with a 5' T7 promoter sequence using HiScibe T7 Quick High yield RNA Synthesis kit (NEB). The transcription was performed at $37^{\circ} \mathrm{C}$ overnight and then purified by phenol: chloroform extraction, followed by ethanol precipitation. Purified sgRNA was quantified by spectrometry and stored at $-80^{\circ} \mathrm{C}$.

\section{In vitro cleavage assay}

Cas9 protein and transcribed sgRNA were incubated for $10 \mathrm{~min}$ at room temperature in reaction buffer containing $1 \times$ NEB buffer 3.1 (NEB Biolabs) supplemented with $1 \mathrm{mM}$ DTT to form Cas9-sgRNA RNP complex. Cleavage was performed in $10 \mu \mathrm{L}$ reactions containing $100 \mathrm{ng}$ of substrate DNA and $1 \mu \mathrm{L}$ RNP complex of indicated concentrations at room temperature for $1 \mathrm{~h}$. Reactions were terminated by addition of $1 \times$ DNA loading buffer and resolved on $2 \%$ agarose gels. For inhibition experiments, G8P $P_{P D}$ peptides (GeneScript, Nanjing, China) were dissolved in deionized distilled water and incubated with Cas9 protein or pre-assembled Cas9-sgRNA RNP for $10 \mathrm{~min}$ at room temperature and the mixed solution was then added to the in vitro cleavage reaction. For 
experiments comparing the inhibitory activities of G8 $\mathrm{P}_{\mathrm{PD}}$ peptides, $0.1 \%$ dimethyl sulfoxide (DMSO) was included in the reaction solution to solubilize lyophilized peptide samples. His-tagged full-length G8P had poor expression in BL21 (DE3) E. coli when expressed from pET28a vector and was thus excluded from the in vitro analyses.

\section{Electrophoresis mobility shift assay (EMSA)}

sgRNA concentration is fixed to $15 \mu \mathrm{M}$ and Cas9 protein was titrated with a molar ratio of Cas9 over sgRNA ranging from 0.05 to 1 . f1 $\mathrm{G}_{8 \mathrm{PD}}$ of 150,300 , and $600 \mu \mathrm{M}$ was incubated with Cas9 protein or pre-assembled Cas9sgRNA RNP complex for $20 \mathrm{~min}$ at $25^{\circ} \mathrm{C}$ and quenched by addition of $1 \times$ native DNA loading buffer containing $40 \mathrm{mM}$ Tris, $\mathrm{pH} 8.2,40 \mathrm{mM}$ acetate, $1 \mathrm{mM}$ ethylenediaminetetraacetic acid (EDTA), $12.5 \%$ (v/v) glycerol, $0.025 \%(\mathrm{~m} / \mathrm{v})$ bromophenol blue. The samples were run on $2 \%$ agarose gels. For the order-of-addition experiment, Cas9 and sgRNA concentrations are fixed to 7.5 and $15 \mu \mathrm{M}$ respectively. SpCas9 was pre-incubated with sgRNA or $\mathrm{G}_{8 \mathrm{PD}}$ for $20 \mathrm{~min}$, followed by incubation with the counterpart for $20 \mathrm{~min}$ at $25^{\circ} \mathrm{C}$.

\section{Chemical crosslinking and mass spectrometry}

Cas9 protein and M13 G8 $\mathrm{P}_{\mathrm{PD}}$ were crosslinked using collision-induced dissociation (CID)-cleavable crosslinkerdisuccinimido sulfoxide (DSSO) following the described procedure [55] with minor modification. Cas9 protein and peptides were mixed in PBS and incubated for $30 \mathrm{~min}$ at room temperature. Crosslinking was performed for 30 min by adding DSSO (Thermo Scientific) to protein/peptide solution with $1000 \mathrm{M}$ excess. The crosslinking reaction was quenched by excess Tris and the crosslinked products were digested with chymotrypsin. The LC MSn data of digested peptides were collected on Orbitrap Fusion Tribrid (Thermo Scientific) with an on-line NanoLC system and analyzed using CID-MS ${ }^{2}-\mathrm{MS}^{3}$ strategy as previously described [62]. Monoisotopic mass of parent ions and corresponding fragment ions, parent ion charge states, and ion intensities from LC MS ${ }^{2}$ and $\mathrm{LC} \mathrm{MS}^{3}$ spectra were extracted using Xcalibur v 3.0 (Thermo Scientific). Database searching was performed using Proteome Discoverer v 2.2 software (Thermo Scientific). Chymotrypsin was set as the enzyme with two missed cleavages being allowed as the maximum values. Protein N-terminal acetylation, methionine oxidation $(15.995 \mathrm{Da})$, carbamidomethyl cysteine $(57.021 \mathrm{Da})$, hydrolyzed lysine DSSO (176.014 Da), and lysine DSSO TRIS $(279.078 \mathrm{Da})$ were selected as variable modifications. In addition, to account for the residual crosslinker, three defined modifications on uncleaved lysines were chosen including alkene $\left(\mathrm{C}_{3} \mathrm{H}_{2} \mathrm{O}\right.$, $54 \mathrm{Da})$, sulfenic acid $\left(\mathrm{C}_{3} \mathrm{H}_{4} \mathrm{O}_{2} \mathrm{~S}, 104 \mathrm{Da}\right)$ and thiol $\left(\mathrm{C}_{3} \mathrm{H}_{2} \mathrm{SO}, 86 \mathrm{Da}\right)$ modifications. A false discovery rate
(FDR) of $1 \%$ was employed to filter out false positive results. The MS, $\mathrm{MS}^{2}$, and $\mathrm{MS}^{3}$ mass tolerances were set as $10 \mathrm{ppm}, 20 \mathrm{ppm}$, and $0.6 \mathrm{Da}$ respectively.

The XlinkX detect program (Thermo Scientific) was used to search $\mathrm{MS}^{2}$ data and identify the list of putative DSSO-interlinked products based on their unique DSSO fragmentation patterns. Monoisotopic masses and charges of parent ions measured in $\mathrm{MS}^{3}$ scans for those putative crosslinked peptides were further validated and scored by XlinkX. The final results were confirmed by manual inspection of the $\mathrm{MS}^{2}$ and $\mathrm{MS}^{3}$ spectra, respectively.

\section{Circular dichroism}

Circular dichroism spectroscopy (Chirascan-plus CD spectrometer, Applied Photophysics) was done to probe the peptide conformational changes. One hundredmicromolar WT and mutant 2 G8P $\mathrm{P}_{\mathrm{PD}}$ peptides were dissolved in deionized water. The $\mathrm{CD}$ data were recorded at $25^{\circ} \mathrm{C}$ and the average value of three biological replicates was presented.

\section{Inhibition of CRISPR-Cas9 activity in human cells by $\mathrm{GBP}_{\mathrm{PD}}$ overexpression}

K562 cells $\left(2 \times 10^{5}\right)$ were harvested, washed with PBS and re-suspended in $20 \mu \mathrm{L}$ of SF nucleofection buffer

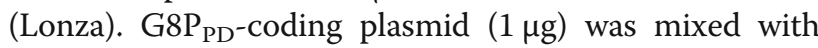
re-suspended K562 cells and nucleofected by Lonza 4D nucleofector with program FF-120. Immediately following the nucleofection, $100 \mu \mathrm{L}$ pre-warmed RPMI-1640 medium was added into nucleofection cuvettes, and the cells were transferred to culture dishes. At 24 post nucleofection, plasmids encoding SpCas9 (500 ng) and AAVS1 or EMX1-targeting sgRNA (250 ng) (Additional file 2: Table S1) were transfected into $\mathrm{G}_{\mathrm{P}} \mathrm{P}_{\mathrm{PD}}-\mathrm{ex}-$ pressing K562 cells by nucleofection as described above.

Low-passage HEK293T cells were seeded into 24-well plates at a density of 150,000 cells per well. The next day, G8P plasmid $(1 \mu \mathrm{g})$ were transfected into cells using Lipofectamine 3000 (Invitrogen). At $24 \mathrm{~h}$ post G8P transfection, plasmids encoding SpCas9 $(0.5 \mu \mathrm{g})$ and AAVS1-targeting sgRNA (250 ng) were co-transfected into G8P-expressing HEK293T cells using lipofectamine.

For G8P pre-incubation experiments, sgRNA-expressing and G8P-expressing plasmids contain green fluorescent protein (GFP) and mCherry reporters, respectively, the expression of which are under the control of promoters independent of sgRNA or G8P expression. For the mock groups without G8P expression, an empty mCherryexpressing plasmid was transfected to control for cell stress. At least 2000 mCherry and GFP dual positive cells were collected using a BD FACSAria III flow cytometer (BD Biosciences) for subsequent analyses. For cotransfection experiments, K562 and Hela cells were nucleofected with $1 \mu \mathrm{g}$ of G8P plasmid, $0.5 \mu \mathrm{g}$ of SpCas9 
plasmid, and $0.5 \mu \mathrm{g}$ sgRNA plasmid. At $48 \mathrm{~h}$ after transfection, unsorted cells were collected for subsequent analyses.

For NmCas9 and SaCas9 experiments, low-passage HEK293T cells were seeded into 48-well plates at a density of 100,000 cells per well. The next day, f1 G8P $\mathrm{P}_{\mathrm{PD}}$ plasmid $(1.5 \mu \mathrm{g})$ were transfected into cells using Lipofectamine 3000 (Invitrogen). At $24 \mathrm{~h}$ post $\mathrm{G}_{8 \mathrm{PD}}$ transfection, plasmids encoding SaCas9 $(0.75 \mu \mathrm{g})$ and $E M X 1$ targeting sgRNA or FANCY-targeting sgRNA $(0.375 \mu \mathrm{g})$ were co-transfected into G8P $\mathrm{PD}^{-}$-expressing HEK293T cells. For NmeCas9, plasmids encoding NmeCas9 $(0.75 \mu \mathrm{g})$ and LINC01588-targeting sgRNA $(0.375 \mu \mathrm{g})$ were co-transfected into $\mathrm{G}_{8} \mathrm{P}_{\mathrm{PD}^{-}}$-expressing HEK293T cells. At $48 \mathrm{~h}$ after transfection of SaCas9 or NmeCas9 and sgRNA plasmids, mCherry and GFP dual positive cells were collected as described above for subsequent analyses.

The genomic DNA of collected cells was extracted using QuickExtract DNA Extraction Solution (Epicentre). Genomic PCR reaction was performed using $100 \mathrm{ng}$ genomic DNA, corresponding primers (Additional file 2: Table S2), Phanta Max Super-fidelity DNA Polymerase (Vazyme), or KOD plus (Takara) using a touchdown cycling protocol $\left(30\right.$ cycles of $98^{\circ} \mathrm{C}$ for $10 \mathrm{~s}$, $68-58^{\circ} \mathrm{C}$ for $15 \mathrm{~s}$ and $68^{\circ} \mathrm{C}$ for $\left.60 \mathrm{~s}\right)$. The PCR products were digested by T7E1 enzyme (NEB), resolved on $2 \%$ agarose gel and then analyzed by densitometry measurements as described [63]. Two or three biological replicates were performed for each condition.

The sgRNA sequences used in this study are as follows (PAM sequence uppercased): SpCas9-CCR5 (tgacatcaattattatacatCGG; for in vitro cleavage reaction), SpCas9EMX1 (gagtccgagcagaagaagaaGGG), SpCas9-AAVS1 (gggagggagagcttggcaggGGG), SpCas9-HBB (cttgcccca cagggcagtaaCGG), NmCas9-LINC01588 (cgcaaagctgcatc cacccccgAGACC), SaCas9-EMX1 (gcaaccacaaacccacga gggCAGAGT), SaCas9-FANCY (gcaaggcccggcgcacggtgg CGGGGT).

\section{Next-generation sequencing of edited genomic sites}

Genomic DNA (100 ng) from AAVS1-edited K562 cells was subject to PCR reactions using stubbed primers (Additional file 2: Table S2). PCR products were purified using Gel Extraction Kit (OMEGA). A high-throughput library preparation kit (Hiseq3000 SBS\&Cluster kit) was used to generate dual-indexed sequence. Two or three biological replicates were processed by Genergy Biotech (Shanghai, China) or Genewiz (Suzhou, Jiangsu, China) using Illumina HiSeq 3000 platform.

Next-generation sequencing library preparations were constructed following the manufacturer's protocol (VAHTS Universal DNA Library Prep Kit, Illumina). For each sample, more than $50 \mathrm{ng}$ purified PCR fragment was used for direct library preparation. The fragments were treated with End Prep Enzyme Mix for end repairing, $5^{\prime}$ phosphorylation and dA-tailing in one reaction, followed by a T-A ligation to add adaptors to both ends. Size selection of adaptor-ligated DNA was then performed using VAHTSTM DNA Clean Beads. Each sample was then amplified by PCR for 8 cycles using P5 and P7 primers. Both P5 and P7 primers carry sequences that can anneal with flowcell to perform bridge PCR. In addition, P7 primer carries a six-base index allowing for multiplexing. The PCR products were cleaned up using VAHTSTM DNA Clean Beads, validated using an Agilent 2100 Bioanalyzer (Agilent Technologies, Palo Alto, CA, USA) and quantified by Qubit2.0 Fluorometer (Invitrogen, Carlsbad, CA, USA). Then libraries with different indexes were multiplexed and loaded on an Illumina HiSeq instrument according to the manufacturer's instructions (Illumina, San Diego, CA, USA). Sequencing was carried out using a $2 \times 150$ paired-end (PE) configuration. Image analyses and base calling were conducted by the HiSeq Control Software (HCS) + OLB + GAPipeline-1.6 (Illumina) on the HiSeq instrument. Sequencing reads were obtained in the Fastq format.

Amplicons with less than $6 \mathrm{M}$ read counts were excluded from the analyses. Short reads were aligned to the reference sequence by Bowtie2 [64] with the following parameters: -D 5 -R 3 -N 1 --gbar 1 --rdg 5,1 --rfg 5, 1 --dovetail. Aligned reads were sorted by SAMtools [65], and INDEL and SNP calling was performed by mpileup [66] with maximum read depth per sample equal to the total reads mapped. VarScan v2.4 [67] was used for the quality control of SNPs and INDELs in mpileup output with a minimum variant frequency of $\geq 0.001$, and a $P$ value threshold of $\leq 0.05$. With the above settings, the following items were quantified including the proportions of reads with INDELs/SNPs at each position in the 20-bp target region, the proportions of INDEL with different insertion or deletion length, the proportions of INDEL reading frames $(3 \mathrm{~N}, 3 \mathrm{~N}+1$, and $3 \mathrm{~N}+2)$, and the proportions of reads harboring variants over the total number of aligned reads.

\section{ChIP-qPCR analyses of the effects of G8Ps on dCas9 binding}

For ChIP experiments, low-passage Hela cells were seeded on to $15-\mathrm{cm}$ plates at a density of $4 \times 10^{6}$ cells per plate. The next day, a total of $40.5 \mu \mathrm{g} \mathrm{M} 13 \mathrm{G} \mathrm{P}_{\mathrm{PD}}$ or

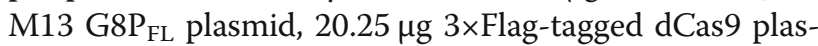
mid, and $20.25 \mu \mathrm{g}$ sgRNA plasmid were transfected into cells using Lipofectamine 3000 (Invitrogen). Two biological replicates were performed for each condition. ChIP was performed with Simple ChIP Enzymatic Chromatin IP Kit (Cell Signaling Technology) following the 
manufacturer's instructions. After $48 \mathrm{~h}$, the cells were washed once with PBS, and $1.25 \mathrm{~mL}$ of $16 \%$ formaldehyde (Life Technologies) was added to $20 \mathrm{~mL}$ of serum-free DMEM for crosslinking to a final formaldehyde concentration of $1 \%$. After $10 \mathrm{~min}$ of crosslinking at room temperature, the cells were harvested for the ChIP assay. The chromatin was sonicated using Branson digital sonifier for 10 cycles of "on/off" pulse with a 30-s interval. Fragmented chromatin was diluted and incubated with $2 \mu \mathrm{g}$ anti-Flag Chip grade antibody (Sigma, USA) overnight at $4{ }^{\circ} \mathrm{C}$. qPCR with immunoprecipitation-purified DNA as the template was used to analyze the effects of G8Ps on dCas9 binding at the on-target and off-target sites.

\section{Statistical analyses}

Two or three biological replicates were performed for each experimental condition. Significant difference was analyzed using one-way ANOVA with Dunnett's multiple comparisons test unless otherwise noted.

\section{Supplementary information}

Supplementary information accompanies this paper at https://doi.org/10. 1186/s13059-020-01956-x.

Additional file 1: Figure S1. Comparison of the in vitro activity of M13 and $f 1$ phage G8P $P_{P D}$. Figure S2. MS analyses of the interface between SpCas9 and M13 G8P SpCas9 K1158 and K1176 mutants. Figure S4. Profile of SpCas9-induced mutations in the absence and presence of G8P PD. Figure S5. G8P PD peptides derived from inoviridae bacteriophages. Figure S6. NGS analyses of the effects of G8P pre-incubation on NmCas9 and SaCas9 in HEK293 cells. Figure S7. NGS analyses of the effects of G8Ps on the specificity of SpCas9 at HBB site in HEK293 cells. Figure S8. Cytotoxicity of CRISPR inhibitors. Figure S9. ChIP-qPCR analyses of the effects of M13 G8Ps on Cas9 binding at AAVS1 on target and pre-determined off-target sites in Hela cells.

Additional file 2: Table S1. sgRNA-targeted genomic sites in this study. Table S2. Primer list.

Additional file 3. Review history.

\section{Acknowledgements}

We thank the Analytical Platform and High-Throughput Screening Platform at Shanghai Institute for Advanced Immunochemical Studies (SIAIS) at ShanghaiTech University for the support of mass spectrometry and flow cytometry experiments.

\section{Peer review information}

Yixin Yao was the primary editor on this article and managed its editorial process and peer review in collaboration with the rest of the editorial team.

\section{Review history}

The review history is available as Additional file 3.

\section{Funding}

This work is supported by the National Natural Science Foundation of China (31600686 to J.L. and 31500632 and U19A2011 to P.M.) and ShanghaiTech University Startup Fund (2019F0301-000-01 to J.L.).

\section{Availability of data and materials}

The mass spectrometry data have been deposited into ProteomeXchange with the accession number PXD012466 [68]. Deep sequencing data have been deposited into NCBI SRA database with the accession number
SRP180801 [69] and SRP199555 [70]. Additional data that support the findings of this study are available upon request from the lead contact author J. Liu.

\section{Authors' contributions}

P.M. and J.L. conceptualized the study. P.M., J.L., Y.-R.C., and S.-J.W. designed the experiments and analyzed data. Y.-R.C. and S.-J.W. performed the in vitro and in vivo Cas9-inhibiting experiments. J.C. analyzed next-generation sequencing data. J. Li manufactured M13 phage and initial phage selections. P.M. and S. W performed the crosslink experimenting. W.C. and W.Z. performed MS experiment and analyzed the data. B.M. and B.Y. provided purified WT SpCas9 proteins and advice on the purification of other SpCas9 variants. S.W. and Z.Z. helped to express and purify SpCas9 proteins. B.J. and G.Y. provided critical resources. P.M. and J.L. wrote the manuscript. All authors discussed the results and commented on and approved the manuscript.

Ethics approval and consent to participate

Not applicable.

Consent for publication

Not applicable.

\section{Competing interests}

The authors declare that they have no competing interests.

\section{Author details}

'Shanghai Institute for Advanced Immunochemical Studies, ShanghaiTech University, Shanghai 201210, China. ${ }^{2}$ School of Life Science and Technology, ShanghaiTech University, Shanghai, China. ${ }^{3} \mathrm{CAS}$ Center for Excellence in Molecular Cell Science, Shanghai Institute of Biochemistry and Cell Biology, Chinese Academy of Sciences, Shanghai, China. ${ }^{4}$ University of Chinese Academy of Sciences, Beijing, China. ${ }^{5}$ College of Life Sciences, Zhejiang University, Hangzhou 310058, Zhejiang, China. ${ }^{6}$ School of Pharmacy, Key Laboratory of Molecular Pharmacology and Drug Evaluation (Yantai University), Ministry of Education, Collaborative Innovation Center of Advanced Drug Delivery System and Biotech Drugs in Universities of Shandong, Yantai University, 264005 Yantai, Shandong, People's Republic of China.

Received: 26 August 2019 Accepted: 10 February 2020

Published online: 26 February 2020

\section{References}

1. Barrangou R, Fremaux C, Deveau H, Richards M, Boyaval P, Moineau S, et al. CRISPR provides acquired resistance against viruses in prokaryotes. Science. 2007:315:1709-12

2. Brouns SJ, Jore MM, Lundgren M, Westra ER, Slijkhuis RJ, Snijders AP, et al. Small CRISPR RNAs guide antiviral defense in prokaryotes. Science. 2008:321:960-4.

3. Marraffini LA, Sontheimer EJ. CRISPR interference limits horizontal gene transfer in staphylococci by targeting DNA. Science. 2008;322:1843-5.

4. Jinek M, Chylinski K, Fonfara I, Hauer M, Doudna JA, Charpentier E. A programmable dual-RNA-guided DNA endonuclease in adaptive bacterial immunity. Science. 2012;337:816-21.

5. Jinek M, East A, Cheng A, Lin S, Ma E, Doudna J. RNA-programmed genome editing in human cells. Elife. 2013;2:e00471.

6. Mali P, Yang L, Esvelt KM, Aach J, Guell M, DiCarlo JE, et al. RNA-guided human genome engineering via Cas9. Science. 2013;339:823-6.

7. Cong L, Ran FA, Cox D, Lin S, Barretto R, Habib N, et al. Multiplex genome engineering using CRISPR/Cas systems. Science. 2013;339:819-23.

8. Cho SW, Kim S, Kim JM, Kim JS. Targeted genome engineering in human cells with the Cas9 RNA-guided endonuclease. Nat Biotechnol. 2013;31:230-2.

9. Koo T, Lee J, Kim JS. Measuring and reducing off-target activities of programmable nucleases including CRISPR-Cas9. Mol Cells. 2015;38:475-81.

10. Kosicki M, Tomberg K, Bradley A. Repair of double-strand breaks induced by CRISPR-Cas9 leads to large deletions and complex rearrangements. Nat Biotechnol. 2018:36:765-71.

11. Li C, Psatha N, Gil S, Wang H, Papayannopoulou T, Lieber A. HDAd5/35(++) adenovirus vector expressing anti-CRISPR peptides decreases CRISPR/Cas9 toxicity in human hematopoietic stem cells. Mol Ther Methods Clin Dev. 2018;9:390-401 
12. Hsu PD, Scott DA, Weinstein JA, Ran FA, Konermann S, Agarwala V, et al. DNA targeting specificity of RNA-guided Cas9 nucleases. Nat Biotechnol. 2013;31:827-32.

13. Pattanayak V, Lin S, Guilinger JP, Ma E, Doudna JA, Liu DR. High-throughput profiling of off-target DNA cleavage reveals RNA-programmed Cas9 nuclease specificity. Nat Biotechnol. 2013;31:839-43.

14. Fu Y, Sander JD, Reyon D, Cascio VM, Joung JK. Improving CRISPR-Cas nuclease specificity using truncated guide RNAs. Nat Biotechnol. 2014;32:279-84.

15. Fellmann C, Gowen BG, Lin PC, Doudna JA, Corn JE. Cornerstones of CRISPR-Cas in drug discovery and therapy. Nat Rev Drug Discov. 2017;16:89-100.

16. Cao J, Wu L, Zhang SM, Lu M, Cheung WK, Cai W, et al. An easy and efficient inducible CRISPR/Cas9 platform with improved specificity for multiple gene targeting. Nucleic Acids Res. 2016;44:e149.

17. Nihongaki Y, Kawano F, Nakajima T, Sato M. Photoactivatable CRISPR-Cas9 for optogenetic genome editing. Nat Biotechnol. 2015;33:755-60.

18. Nunez JK, Harrington LB, Doudna JA. Chemical and biophysical modulation of Cas9 for tunable genome engineering. ACS Chem Biol. 2016;11:681-8.

19. Wright AV, Sternberg SH, Taylor DW, Staahl BT, Bardales JA, Kornfeld JE, et al. Rational design of a split-Cas9 enzyme complex. Proc Natl Acad Sci U S A. 2015;112:2984-9.

20. Maji B, Gangopadhyay SA, Lee M, Shi M, Wu P, Heler R, et al. A highthroughput platform to identify small-molecule inhibitors of CRISPR-Cas9. Cell. 2019;177:1067-79.

21. Pawluk A, Davidson AR, Maxwell KL. Anti-CRISPR: discovery, mechanism and function. Nat Rev Microbiol. 2018;16:12-7.

22. Stanley SY, Maxwell KL. Phage-encoded anti-CRISPR defenses. Annu Rev Genet. 2018:52:445-64.

23. Bondy-Denomy J, Pawluk A, Maxwell KL, Davidson AR. Bacteriophage genes that inactivate the CRISPR/Cas bacterial immune system. Nature. 2013;493:429-32.

24. Bondy-Denomy J, Davidson AR, Doudna JA, Fineran PC, Maxwell KL, Moineau $S$, et al. A unified resource for tracking anti-CRISPR names. CRISPR J. 2019;1:304-5.

25. Pawluk A, Bondy-Denomy J, Cheung VH, Maxwell KL, Davidson AR. A new group of phage anti-CRISPR genes inhibits the type I-E CRISPR-Cas system of Pseudomonas aeruginosa. MBio. 2014;5:e00896.

26. Pawluk A, Staals RH, Taylor C, Watson BN, Saha S, Fineran PC, et al. Inactivation of CRISPR-Cas systems by anti-CRISPR proteins in diverse bacterial species. Nat Microbiol. 2016;1:16085.

27. He F, Bhoobalan-Chitty Y, Van LB, Kjeldsen AL, Dedola M, Makarova KS, et al. Anti-CRISPR proteins encoded by archaeal lytic viruses inhibit subtype I-D immunity. Nat Microbiol. 2018;3:461-9.

28. Marino ND, Zhang JY, Borges AL, Sousa AA, Leon LM, Rauch BJ, et al. Discovery of widespread type I and type V CRISPR-Cas inhibitors. Science. 2018;362:240-2.

29. Pawluk A, Amrani N, Zhang Y, Garcia B, Hidalgo-Reyes Y, Lee J, et al. Naturallyoccurring off-switches for CRISPR-Cas9. Cell. 2016;167:1829-38. e1829.

30. Hynes AP, Rousseau GM, Lemay ML, Horvath P, Romero DA, Fremaux C, et al. An anti-CRISPR from a virulent streptococcal phage inhibits streptococcus pyogenes Cas9. Nat Microbiol. 2017:2:1374-80.

31. Rauch BJ, Silvis MR, Hultquist JF, Waters CS, McGregor MJ, Krogan NJ, et al. Inhibition of CRISPR-Cas9 with bacteriophage proteins. Cell. 2017;168:150-8. e110.

32. Uribe RV, van der Helm E, Misiakou MA, Lee SW, Kol S, Sommer MOA Discovery and characterization of Cas 9 inhibitors disseminated across seven bacterial phyla. Cell Host Microbe. 2019;25:233-41. e235.

33. Watters KE, Fellmann C, Bai HB, Ren SM, Doudna JA. Systematic discovery of natural CRISPR-Cas12a inhibitors. Science. 2018;362:236-9.

34. Lee J, Mir A, Edraki A, Garcia B, Amrani N, Lou HE, et al. Potent Cas9 inhibition in bacterial and human cells by AcrllC4 and AcrllC5 anti-CRISPR proteins. MBio. 2018;9.

35. Basgall EM, Goetting SC, Goeckel ME, Giersch RM, Roggenkamp E, Schrock MN, et al. Gene drive inhibition by the anti-CRISPR proteins AcrllA2 and AcrllA4 in Saccharomyces cerevisiae. Microbiology. 2018;164: 464-74

36. Harrington LB, Doxzen KW, Ma E, Liu JJ, Knott GJ, Edraki A, et al. A broadspectrum inhibitor of CRISPR-Cas9. Cell. 2017:170:1224-33. e1215.

37. Hynes AP, Rousseau GM, Agudelo D, Goulet A, Amigues B, Loehr J, et al. Widespread anti-CRISPR proteins in virulent bacteriophages inhibit a range of Cas9 proteins. Nat Commun. 2018;9:2919.
38. Shin J, Jiang F, Liu JJ, Bray NL, Rauch BJ, Baik SH, et al. Disabling Cas9 by an anti-CRISPR DNA mimic. Sci Adv. 2017:3:e1701620.

39. Li J, Xu Z, Chupalov A, Marchisio MA. Anti-CRISPR-based biosensors in the yeast S. cerevisiae. J Biol Eng. 2018;12:11.

40. Nakamura M, Srinivasan P, Chavez M, Carter MA, Dominguez AA, La Russa $M$, et al. Anti-CRISPR-mediated control of gene editing and synthetic circuits in eukaryotic cells. Nat Commun. 2019;10:194.

41. Jiang F, Liu JJ, Osuna BA, Xu M, Berry JD, Rauch BJ, et al. Temperatureresponsive competitive inhibition of CRISPR-Cas9. Mol Cell. 2019;73:601-10. e605.

42. Bubeck F, Hoffmann MD, Harteveld Z, Aschenbrenner S, Bietz A, Waldhauer $M C$, et al. Engineered anti-CRISPR proteins for optogenetic control of CRISPR-Cas9. Nat Methods. 2018:15:924-7.

43. Hoffmann MD, Aschenbrenner S, Grosse S, Rapti K, Domenger C, Fakhiri J, et al. Cell-specific CRISPR-Cas9 activation by microRNA-dependent expression of anti-CRISPR proteins. Nucleic Acids Res. 2019;47:e75

44. Palmer DJ, Turner DL, Ng P. Production of CRISPR/Cas9-mediated selfcleaving helper-dependent adenoviruses. Mol Ther Methods Clin Dev. 2019;13:432-9.

45. Dong GM, Wang S, Zhu Y, Xiong Z, Yang J, et al. Structural basis of CRISPRSpyCas9 inhibition by an anti-CRISPR protein. Nature. 2017:546:436-9.

46. Yang H, Patel DJ. Inhibition mechanism of an anti-CRISPR suppressor AcrllA4 targeting SpyCas9. Mol Cell. 2017:67:117-27. e115.

47. Wang X, Yao D, Xu JG, Li AR, Xu J, Fu P, et al. Structural basis of Cas3 inhibition by the bacteriophage protein AcrF3. Nat Struct Mol Biol. 2016;23:868-70

48. Wang J, Ma J, Cheng Z, Meng X, You L, Wang M, et al. A CRISPR evolutionary arms race: structural insights into viral anti-CRISPR/Cas responses. Cell Res. 2016;26:1165-8.

49. Dong L, Guan X, Li N, Zhang F, Zhu Y, Ren K, et al. An anti-CRISPR protein disables type $V$ Cas12a by acetylation. Nat Struct Mol Biol. 2019; 26:308-14.

50. Barbas CF 3rd, Kang AS, Lerner RA, Benkovic SJ. Assembly of combinatorial antibody libraries on phage surfaces: the gene III site. Proc Natl Acad Sci U S A. 1991;88:7978-82.

51. Smith GP, Petrenko VA. Phage display. Chem Rev. 1997:97:391-410.

52. van Wezenbeek PM, Hulsebos TJ, Schoenmakers JG. Nucleotide sequence of the filamentous bacteriophage M13 DNA genome: comparison with phage fd. Gene. 1980;11:129-48.

53. Marvin DA. Filamentous phage structure, infection and assembly. Curr Opin Struct Biol. 1998:8:150-8

54. Morag O, Sgourakis NG, Baker D, Goldbourt A. The NMR-Rosetta capsid model of M13 bacteriophage reveals a quadrupled hydrophobic packing epitope. Proc Natl Acad Sci U S A. 2015;112:971-6.

55. Kao A, Chiu CL, Vellucci D, Yang Y, Patel VR, Guan S, et al. Development of a novel cross-linking strategy for fast and accurate identification of crosslinked peptides of protein complexes. Mol Cell Proteomics. 2011:10:M110 002212

56. Nishimasu H, Ran FA, Hsu PD, Konermann S, Shehata SI, Dohmae N, et al. Crystal structure of Cas9 in complex with guide RNA and target DNA. Cell. 2014;156:935-49.

57. Zhu Y, Gao A, Zhan Q, Wang Y, Feng H, Liu S, et al. Diverse mechanisms of CRISPR-Cas9 inhibition by type IIC anti-CRISPR proteins. Mol Cell. 2019;74:296-309. e297.

58. Antoniani C, Meneghini V, Lattanzi A, Felix T, Romano O, Magrin E, et al. Induction of fetal hemoglobin synthesis by CRISPR/Cas9-mediated editing of the human beta-globin locus. Blood. 2018;131:1960-73.

59. Bondy-Denomy J, Garcia B, Strum S, Du M, Rollins MF, Hidalgo-Reyes Y, et al. Multiple mechanisms for CRISPR-Cas inhibition by anti-CRISPR proteins. Nature. 2015;526:136-9.

60. Jinek M, Jiang F, Taylor DW, Sternberg SH, Kaya E, Ma E, et al. Structures of Cas9 endonucleases reveal RNA-mediated conformational activation. Science. 2014;343:1247997

61. Li B, Zeng C, Li W, Zhang X, Luo X, Zhao W, et al. Synthetic oligonucleotides inhibit CRISPR-Cpf1-mediated genome editing. Cell Rep. 2018;25:3262-72. e3263.

62. Liu F, LossI P, Scheltema R, Viner R, Heck AJR. Optimized fragmentation schemes and data analysis strategies for proteome-wide cross-link identification. Nat Commun. 2017:8:15473.

63. Guschin DY, Waite AJ, Katibah GE, Miller JC, Holmes MC, Rebar EJ. A rapid and general assay for monitoring endogenous gene modification. Methods Mol Biol. 2010;649:247-56 
64. Langmead B, Salzberg SL. Fast gapped-read alignment with Bowtie 2. Nat Methods. 2012;9:357-9.

65. Li H, Handsaker B, Wysoker A, Fennell T, Ruan J, Homer N, et al. The sequence alignment/map format and SAMtools. Bioinformatics. 2009;25:2078-9.

66. Li H, Durbin R. Fast and accurate short read alignment with BurrowsWheeler transform. Bioinformatics. 2009;25:1754-60.

67. Koboldt DC, Zhang Q, Larson DE, Shen D, McLellan MD, Lin L, et al. VarScan 2: somatic mutation and copy number alteration discovery in cancer by exome sequencing. Genome Res. 2012;22:568-76.

68. Cui Y, Wang S, Chen J, Jie L, Chen W, Wang S, Meng B, Zhu W, Zhang Z, Yang B , Jiang B, Yang G, Ma P, Liu J. Allosteric inhibition of CRISPR-Cas9 by bacteriophage-derived peptides. Datasets ProteomXchange http:// proteomecentral.proteomexchange.org/cgi/GetDataset?ID=PXD012466 (2020).

69. Cui Y, Wang S, Chen J, Jie L, Chen W, Wang S, Meng B, Zhu W, Zhang Z, Yang B , Jiang B, Yang G, Ma P, Liu J. Allosteric inhibition of CRISPR-Cas9 by bacteriophage-derived peptides. Datasets SequenceReadArchive https://www.ncbi.nlm.nih.gov/sra/SRP180801 (2020).

70. Cui Y, Wang S, Chen J, Jie L, Chen W, Wang S, Meng B, Zhu W, Zhang Z, Yang B , Jiang B, Yang G, Ma P, Liu J. Allosteric Inhibition of CRISPR-Cas9 by Bacteriophage-derived Peptides. Datasets. SequenceReadArchive https://www.ncbi.nlm.nih.gov/sra/SRP199555 (2020).

\section{Publisher's Note}

Springer Nature remains neutral with regard to jurisdictional claims in published maps and institutional affiliations.

Ready to submit your research? Choose BMC and benefit from:

- fast, convenient online submission

- thorough peer review by experienced researchers in your field

- rapid publication on acceptance

- support for research data, including large and complex data types

- gold Open Access which fosters wider collaboration and increased citations

- maximum visibility for your research: over $100 \mathrm{M}$ website views per year

At $\mathrm{BMC}$, research is always in progress.

Learn more biomedcentral.com/submissions 